Meta

Journal des traducteurs

Translators' Journal

\title{
Révision : Définitions et paramètres
}

\section{Hyang Lee}

Volume 51, numéro 2, juin 2006

Théories et pratiques de la traduction et de l'interprétation en Corée

Theories and Practices of Translation and Interpretation in Korea

URI : https://id.erudit.org/iderudit/013265ar

DOI : https://doi.org/10.7202/013265ar

Aller au sommaire du numéro

Éditeur(s)

Les Presses de l'Université de Montréal

ISSN

0026-0452 (imprimé)

1492-1421 (numérique)

Découvrir la revue

Citer cet article

Lee, H. (2006). Révision : Définitions et paramètres. Meta, 51(2), 410-419.

https://doi.org/10.7202/013265ar

\section{Résumé de l'article}

Notre étude a eu pour objectif de réfléchir sur un sujet longtemps négligé par les traductologues malgré son importance primordiale dans tout processus de traduction, à savoir la révision. Dans cet article, il sera d'abord question de la concrétisation du concept. Il s'agira ensuite d'une réflexion sur les diverses définitions du mot qu'ont proposées plusieurs auteurs ainsi que sur les limites montrées par ces derniers quant à apporter une définition claire qui permettrait d'éviter l'écueil d'une confusion terminologique. Finalement, l'objectivité en matière de révision sera abordée, par le biais d'un examen des paramètres proposés par trois auteurs et en vue d'en produire une synthèse pour, en fait, parvenir à un total de quatre paramètres.
Ce document est protégé par la loi sur le droit d'auteur. L'utilisation des services d’Érudit (y compris la reproduction) est assujettie à sa politique d'utilisation que vous pouvez consulter en ligne.

https://apropos.erudit.org/fr/usagers/politique-dutilisation/ 


\title{
Révision : définitions et paramètres
}

\author{
HYANG LEE \\ Université Hankuk des Études étrangères, Séoul, Corée \\ hc612@hanafos.com
}

\begin{abstract}
RÉSUMÉ
Notre étude a eu pour objectif de réfléchir sur un sujet longtemps négligé par les traductologues malgré son importance primordiale dans tout processus de traduction, à savoir la révision. Dans cet article, il sera d'abord question de la concrétisation du concept. II s'agira ensuite d'une réflexion sur les diverses définitions du mot qu'ont proposées plusieurs auteurs ainsi que sur les limites montrées par ces derniers quant à apporter une définition claire qui permettrait d'éviter l'écueil d'une confusion terminologique. Finalement, l'objectivité en matière de révision sera abordée, par le biais d'un examen des paramètres proposés par trois auteurs et en vue d'en produire une synthèse pour, en fait, parvenir à un total de quatre paramètres.
\end{abstract}

\begin{abstract}
Revision has a direct, "one-to-one" relationship with the quality of translation. Nonetheless, the topic has not been sufficiently discussed thus far in the discipline of translation studies. Recognizing the need for further in-depth research on the topic, this study reviews the various discussions related to revision on the basis of the following three categories: First, it explores how the literature has attempted to interpret the concept of revision. Second, it reviews the definitions of revision as suggested by various scholars, at the same time pointing out their limitations and proposing a more clear-cut definition. Lastly, it examines the diverse parameters of revision raised by previous academic endeavors, and based on a comprehensive evaluation and analysis of earlier parameters, suggests four new parameters of revision.
\end{abstract}

\section{초록}

번역의 품질과 직결되어 있는 개념인 감수(revision)는 오늘날까지 번역학자들에 의 해 충분히 연구되지 않은 주제이다. 따라서 본 연구에서는 감수와 관련한 다양한 논의들을 세 가지 차원에서 검토하고자 한다. 우선 감수 개념이 어떤 과정을 통하 여 구체화되어 왔는지를 살펴보았다. 두 번째로는 다양한 학자들이 제시한 감수의 정의 및 그 한계를 짚어보고 이를 통해 명확한 감수 정의를 제시하였다. 마지막으 로 기존 연구에서 제시된 감수의 기준들을 살펴보고, 이를 종합하여 4 개의 감수 기 준을 도출하였다.

\section{MOTS-CLÉS/KEYWORDS}

révision, definition de la révision, paramètres de la révision, objectivité de la révision

\section{Introduction}

Il est souvent dit que le métier de traducteur est l'une des plus anciennes professions du monde et que la révision ne peut être qu'une activité aussi ancienne que la première. Il en faut pour preuve que l'acte consistant à vérifier la fidélité et la lisibilité des textes traduits a toujours été issu de l'acte consistant à traduire.

Bien qu'elle constitue une étape fondamentale du processus de traduction, la révision n'a guère été appréciée à sa juste valeur, jusqu'à présent, par les traductolo- 
gues. Si traduire a fait couler beaucoup d'encre, peu de choses ont, d'un autre côté, été écrites à propos de la deuxième. Le terme même est resté flou, sauf dans quelques pays où prévaut une situation de bilinguisme, voire de multilinguisme, et parmi lesquels on compte le Canada: les impératifs du marché y ont fait naître, dès les années 1970, un vif intérêt pour l'activité qui semble jouer un rôle crucial dans l'amélioration du niveau des traductions.

L'enjeu est donc de réfléchir sur les divers points de vue qui ont concerné jusqu'à nos jours le mot révision afin de savoir comment les discussions ont récemment évolué, afin de dégager une tendance, sinon une direction qui dominerait les débats dans l'avenir.

Nous avons opté pour trois thèmes. En premier lieu, nous nous intéresserons à la concrétisation du concept. Nous nous pencherons ensuite sur les différentes définitions proposées par des auteurs. Nous réfléchirons enfin sur la question de l'objectivité devant être prise en compte dans toute activité de révision et ce à travers différents paramètres.

\section{Concrétisation du concept révision}

\subsection{Nida et l'inflation terminologique}

Nida (1964) tente de théoriser sa longue expérience de traducteur de la Bible et prend déjà conscience de l'importance du travail des réviseurs. En présentant les diverses procédures par lesquelles passent les traducteurs quand ils vérifient si le message original est bien transmis dans la langue cible, le spécialiste nous fait part de ses sentiments sur ce qu'est la révision:

Revisions are in some ways a good deal more difficult than original translations, and hence often involve very complex procedures, usually because of vested interests (p. 245).

Il est évident que cette phrase doit être interprétée en fonction du caractère spécifique que revêt la traduction de la Bible. Étant par nature un immense travail collectif auquel ont toujours participé de nombreux acteurs aux intérêts divers, la traduction des textes sacrés suppose un niveau de coordination et de discussion assez élevé. Nida, toujours en 1964, présente 9 étapes ${ }^{1}$ pour la traduction individuelle et 16 autres ${ }^{2}$ pour la traduction collective. Discuter du contenu de chaque étape et parler de l'efficacité des procédures qui en découlent dépasserait largement le champ de notre étude. Nous ne pourrons cependant pas omettre de mentionner l'extrême variété des termes utilisés par le traductologue vis-à-vis du processus de vérification et de contrôle de la qualité du niveau de traduction, comme studying, scrutiny, revising, polishing, correction ou encore review, pour parler de tous ces travaux qui consistent à retoucher le texte en vue de l'améliorer. Cependant, Nida n'explicite pas de différences entre les termes dont il fait usage, ce qui nous pousse à inférer qu'il les a tous considérés, en quelque sorte, comme des synonymes, à moins que des distinctions, liées à la nuance et dépendant du contexte, nous aient échappé. Toujours est-il que des questions demeurent... Qu'est-ce qui différencie revising et review? Qui ont été les personnes chargées des travaux? Ont-elles toutes été des linguistes qui connaissaient la langue de départ ou des experts du domaine d'étude? Même si le chercheur nous présente par la suite le travail effectué par trois comités ${ }^{3}$, s'attardant même à nous en décrire la 
composition, nous avons du mal à saisir clairement ce qu'il signifie pour chacun des termes employés et chacune des différences sous-entendues dans les idées avancées.

\subsection{Munday et sa "cartographie» de la traductologie}

Si Nida a mentionné la révision comme l'une des nombreuses procédures visant à garantir la qualité de toute traduction, Munday (2001) a, quant à lui, situé le terme comme un champ de recherche de la traductologie.

Ce dernier parle de la révision comme l'un des trois domaines propres à la critique des traductions (Translation criticism). En fait, Munday n'est pas le véritable fondateur de la notion - et il le fait d'ailleurs comprendre dans son ouvre - puisqu'il n'a fait que compléter ce qui a été proposé et interprété avant lui par deux autres auteurs, Holmes et Toury. Holmes (1972) a eu l'idée de classifier pour la première fois les champs de recherches traductologiques en «traductologie pure» et en «traductologie appliquée», ainsi que d'énumérer les sous-domaines des deux branches ainsi mises à jour. Cette classification a par la suite été reprise par Toury (1995) qui l'a complétée et interprétée à sa manière en la visualisant sous la forme d'un arbre, avant que celui-ci ne soit modifié et détaillé de façon plus approfondie par Munday, ce qui nous donne les résultats suivants:

ARBRE 1

Holmes's basic map of translation studies

(Toury 1995: 10 in Munday 2001: 10)

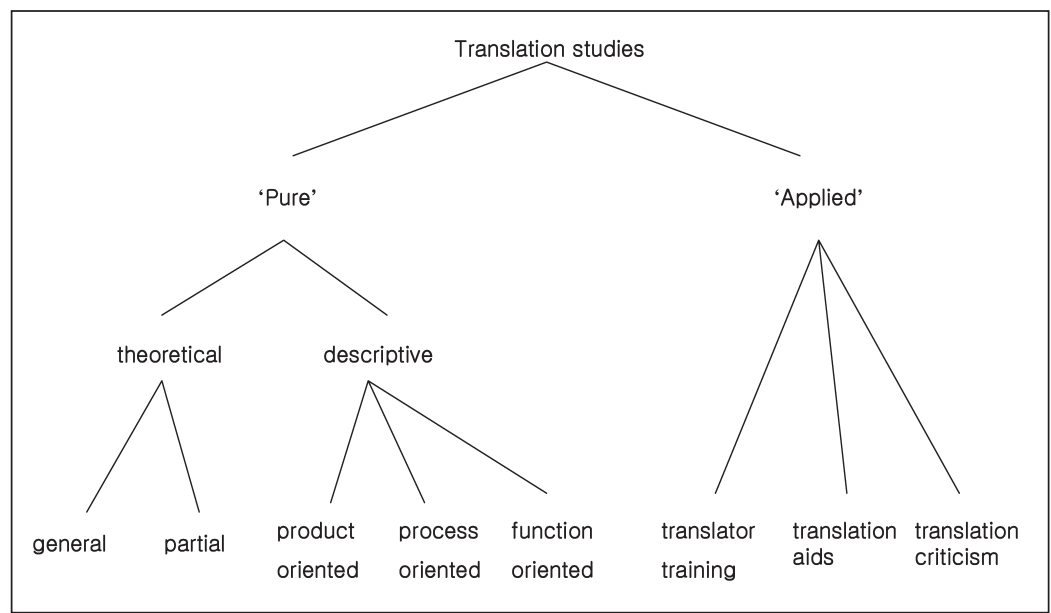

Nous pouvons voir, dans le graphique ci-dessus, que la critique des traductions est mentionnée comme l'un des trois domaines de la traductologie appliquée mais que le mot révision n'y figure pas encore. Munday, désireux d'expliciter davantage, propose un autre graphique encore plus détaillé et consacré entièrement, cette fois-ci, à la traductologie appliquée: 


\section{ARBRE 2}

The applied branch of translation studies in Munday (2001: 13)

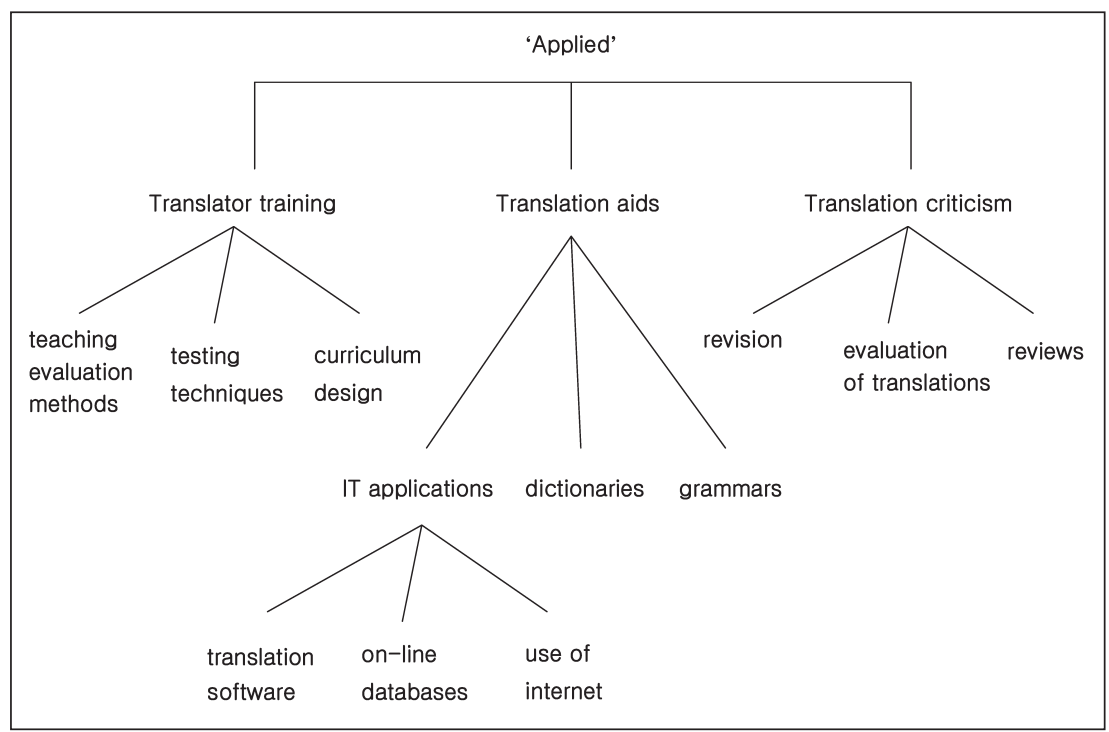

La révision trouve enfin sa place, à côté de l'emploi de reviews et evaluation of translation, en tant que l'un des trois sous-domaines de la critique des traductions.

\section{Quelle définition pour révision?}

La première chose que l'on constate quand on essaye de définir ce qu'est la révision concerne le caractère ambigu et le nombre varié des définitions proposées. En Corée, le terme - gamsu en coréen - désigne essentiellement deux choses fort différentes l'une de l'autre. Il signifie, premièrement, la relecture, qu'effectuent des natifs, de textes traduits du coréen en langue B par des Coréens. Ces correcteurs ne connaissant pas, dans la plupart des cas, la langue de départ - le coréen - leur travail est souvent axé sur la reformulation et la correction des erreurs grammatico-syntaxiques. Le terme signifie également l'examen, effectué par des spécialistes, du contenu des textes traduits et de la terminologie qui y apparait.

Dans l'ouvrage publié par Horguelin et Brunette (1998), la révision est définie comme une activité beaucoup plus vaste et complexe:

La révision se définit comme l'examen attentif d'un texte dans le but de le rendre conforme à des critères linguistiques et fonctionnels reconnus. (p. 3)

On voit clairement qu'ici les auteurs parlent de la révision au sens large du terme: l'objet ne se limite pas aux textes traduits et l'opération peut concerner des textes authentiques. Les auteurs englobent ainsi sous la même dénomination la révision unilingue, qui consiste à garantir la qualité informative et linguistique (contenu et forme) du texte original ou présenté comme tel, et la révision bilingue, qui consiste à faire le même travail pour des textes traduits. Si l'on fait un parallèle avec la situation coréenne, on peut dire que ce qui se fait en Corée est surtout de la révision unilingue 
et que la révision bilingue est presque inexistante. Il est de toute façon indéniable que, dans la pratique, les deux activités se recoupent souvent, ce qui rend encore plus difficile la tâche de les délimiter toutes les deux.

La définition avancée par Graham (1989) trahit également la difficulté de placer une frontière entre les diverses orientations dont le but commun est de retoucher des textes traduits ou originaux. L'auteur essaye donc de mettre en lumière les différences qui séparent le checking, le revising et l'editing mis en œuvre pour assurer aux textes une qualité tout à fait acceptable. L'auteur définit aussi le checking comme une activité pouvant être pratiquée par les non-linguistes et les non-traducteurs, laquelle consiste à corriger les erreurs typographiques, à relever les ambiguités et à vérifier l'orthographe des noms propres. Concernant l'editing, il s'agit surtout de préparer et d'adapter des textes à des fins de publication, d'éliminer les passages non pertinents ou bien d'uniformiser le style. Or, quand Graham tente de définir ce qu'est la révision en énumérant toutes les tâches qui incombent à la discipline - améliorer la terminologie, éclaircir les points obscurs, renforcer les effets, adapter la charge émotive du texte initial à la spécificité du lecteur, assurer la cohérence terminologique, veiller à l'orthographe, à la grammaire et au registre ${ }^{4}$ - on ne cerne plus très bien la distinction entre checking et editing et on a l'impression que le réviseur fait un peu de tout, ce dont l'auteur est conscient:

The duties of the checker and those of the revisor will overlap and both functions may very well be exercised at the same time by the same person. (p. 66).

Si, dans la pratique, le flou dans l'établissement d'une frontière entre les trois activités concernées ne constitue guère d'obstacle à la bonne marche du travail des réviseurs, cela n'est pas toujours le cas sur le plan théorique. Le terme révision doit rester suffisamment univoque pour que nous puissions être sûrs de parler de la même chose et c'est la raison pour laquelle nous avons besoin d'une définition plus concrète apte à différencier la révision des autres termes que sont le checking et l'editing ${ }^{5}$.

Une solution peut être trouvée si l'on prend en compte le profil de la personne chargée de chacune de ces opérations. Si le checking et l'editing peuvent être assurés par des non-linguistes, cela veut dire que les valeurs qu'ils englobent, malgré l'évidente interaction utile et complémentaire qu'ils entretiennent quant à la tâche du réviseur, existent indépendamment des réflexions traductologiques car ciblant des textes autres que des traductions. Il s'ensuit dans cette optique que la révision, au sens traductologique du terme, doit être définie comme une activité qui concerne la traduction et qui doit être assurée par des traducteurs connaissant la langue de départ. D'où la définition suivante du mot révision:

Révision de la traduction (révision bilingue): examen, par un traducteur ou un réviseur connaissant la langue de départ, d'un texte traduit pour le rendre conforme aux besoins et aux attentes du destinataire.

\section{Le problème de l'objectivité dans l'acte de réviser: question de paramètres}

Après nous être mis d'accord sur la définition du mot révision, il nous faut maintenant réfléchir sur un autre aspect du terme dont l'importance est de taille. Comme pour toutes les pratiques d'évaluation, l'acte de réviser des textes traduits par une autre 
personne ne peut être acceptable que si le révisé est convaincu du fait que la révision n'est pas le résultat d'un jugement intuitif ou subjectif de la part du réviseur. Darbelnet résume ainsi la difficulté de se montrer objectif quand on révise une traduction:

Le réviseur est censé posséder une certaine compétence mais qu'est-ce qui prouve qu'il a raison et que si on faisait appel à plusieurs réviseurs ils seraient d'accord? (Darbelnet 1972)

Il n'est donc plus question, pour le réviseur, d'assurer le travail en se montrant intuitif ou impressif. Le problème de l'objectivité dans la tâche de révision nécessite dès lors de poser comme condition préalable l'existence de paramètres adéquats, lesquels ne peuvent cependant pas garantir un caractère objectif absolu à l'activité révisante, un certain degré de subjectivité étant inévitable aussi bien dans le domaine de la révision que dans celui de la traduction.

Avant de présenter et d'analyser les paramètres proposés par les auteurs, il faudrait préliminairement définir ce à quoi le terme renvoie. Horguelin et Brunette (1998) distinguent des principes: si les paramètres sont une réponse à la question "Que faut-il vérifier?», alors les principes sont aussi une autre réponse, celle qui répond à la question «Comment procéder?» (p. 39). Ce qui revient à dire que les paramètres constituent une base du jugement du réviseur, tandis que les principes concernent plutôt l'aspect méthodologique relatif à l'accomplissement des démarches nécessaires à la révision.

Rares sont cependant les spécialistes à avoir proposé des paramètres concrets pour guider le travail du réviseur. Néanmoins, notre nouvelle tâche sera d'examiner et de comparer les paramètres avancés par les trois auteurs que sont Darbelnet, Horguelin et Brunette (fonctionnant en binôme) et Mossop.

\subsection{Les sept paramètres de la révision chez Darbelnet et limites}

Darbelnet (1977) a été l'un des premiers à proposer des paramètres opérationnels pour les réviseurs. Dans son article consacré aux problèmes liés à l'acte de traduire de l'anglais au français, il explicite sept niveaux ou obligations à l'égard du traducteur pour que celui-ci produise une traduction de qualité satisfaisante. Les sept niveaux auxquels doit satisfaire le traducteur sont: 1) le niveau sémantique; 2) le niveau idiomatique; 3) la tonalité de l'original; 4) les faits de culture; 5) les allusions littéraires et folkloriques; 6) les intentions de l'auteur; et 7) le besoin du destinataire. Après avoir expliqué et illustré chaque niveau par des exemples concrets, l'auteur propose d'en tirer les paramètres susceptibles de corriger l'aspect impressif qui trop souvent nuit à la crédibilité des appréciations présentes dans les traductions. Ainsi en propose-t-il sept devant permettre au réviseur de se poser, sur le texte dans lequel il s'investit, un certain nombre de questions précises et abordant chacune un domaine propre (p. 16):

1. Le sens est-il exact, sur les plans global et organique?

2. La langue d'arrivée est-elle idiomatique et astreinte à la propriété des termes?

3. La tonalité est-elle respectée?

4. Les différences de culture sont-elles observées?

5. Les allusions littéraires et folkloriques sont-elles traitées judicieusement?

6. Est-il tenu compte des intentions de l'auteur de façon à ce qu'elles ne s'extériorisent pas dans le discours?

7. La traduction est-elle adaptée à son destinataire? 
Malgré le ton souvent prescriptif qui caractérise les discours traductologiques des premiers temps, l'étude de Darbelnet est d'autant plus significative qu'il est un des rares auteurs à s'être interessé à la révision au point de proposer des paramètres. Il faut pourtant admettre que certains éléments réduisent l'intérêt de sa proposition. Premièrement, les paramètres pris en compte semblent plus facilement applicables à certains textes qu'à d'autres. Les paramètres 4 (faits de culture) et 5 (allusions littéraires et folkloriques) seront pertinents dans le cas des textes littéraires ou culturels mais sans utilité pour des textes plus pragmatiques. L'auteur de l'article le reconnaît d'ailleurs, dans la dernière partie de ses travaux, faisant remarquer que «la grille que l'on pourrait ainsi établir ne serait pleinement utilisée que dans le domaine de la traduction littéraire et de la traduction générale... et que dans le domaine technique, elle se réduirait parfois aux critères sémantique et stylistique» (p. 17). Cela peut-il signifier que nous ayons besoin de paramètres différents selon la nature ou le type de texte impliqué? Il s'agit là d'un tout autre sujet de recherche nécessitant une réflexion approfondie et risquant de dépasser largement le champ de notre étude. Nous nous contenterons de dire que, pour une raison pratique, il est peut-être plus facile d'établir des paramètres suffisamment universels de sorte que ceux-ci ciblent de manière flexible n'importe quel type de texte.

Le deuxième point faible de l'étude de Darbelnet est sa conception même de la traduction. Les nombreux exemples qu'il cite situent souvent ses réflexions sur le plan des microstructures coupées de leur contexte linguistique ou extralinguistique. Par exemple, l'équivalent anglais de sortie de camions serait trucks entering. Son étude regorge d'autres exemples du genre et sa façon d'aborder les choses se reflète finalement dans sa définition des paramètres, lesquels semblent accorder beaucoup plus d'importance à la question de la traduction efficace de certains termes chargés de connotations spécifiques, qu'ils soient littéraires ou folkloriques.

La troisième et dernière remarque porte sur la difficulté de placer une frontière nette entre les paramètres. Les différences de culture (paramètre 3 ) et les allusions littéraires et folkloriques (paramètre 4) ne sont pas si évidentes dans la réalité quotidienne.

\subsection{Horguelin et Brunette}

Si les réflexions de Darbelnet ont principalement porté sur des textes à caractère littéraire, celles de Horguelin et Brunette l'ont été sur des textes à caractère pragmatique en ce qui concerne le champ de la révision. Les paramètres proposés par ces derniers sont: 1) exactitude (fidélité au sens);2) correction (respect du code linguistique); 3 ) lisibilité (facilité de la compréhension); 4) adaptation fonctionnelle (prenant en compte registre, tonalité, destinataire); et 5) rentabilité.

Les deux premiers paramètres reprennent en partie ceux proposés antérieurement par Darbelnet. Nous nous arrêterons sur les trois autres. Tout d'abord, le concept de lisibilité devant permettre d'évaluer la valeur communicationnelle du texte d'arrivée signifie un changement d'approche. Il ne s'agit plus de se soucier des équivalents adéquats de tel ou tel mot mais de savoir si le texte traduit peut être facilement compris par les lecteurs et s'il assure efficacement sa fonction communicative.

Le quatrième paramètre, l'adaptation fonctionnelle, résume bien ce que Darbelnet a voulu exprimer à travers les faits de culture, les allusions littéraires et folkloriques et la prise en compte du destinataire. En englobant tout ceci sous un seul terme plus 
général, qui peut aussi bien être appliqué aux textes littéraires qu'aux textes pragmatiques, Horguelin et Brunette parviennent à proposer un ensemble plus simple et plus clair malgré le nombre réduit des paramètres - cinq au total.

Le cinquième, concernant la rentabilité, est un élément qui n'a pas eu droit au chapitre chez Darbelnet. Il a le mérite de bien présenter l'esprit pragmatique des deux auteurs armés de leur expérience du terrain, lesquels se montrent catégoriques sur les trois points suivants, à savoir que les réviseurs ont le droit de refuser les textes qu'ils estiment médiocres car: 1) l'opération n'est pas rentable; 2) la qualité du produit fini n'est jamais satisfaisante; et 3 ) la réputation du réviseur peut être compromise (p. 37).

Pourtant, bien que le paramètre de lisibilité soit une nouveauté intéressante dans le sens où Horguelin et Brunette abordent les textes sur les plans discursif et global, celui concernant la rentabilité reste un concept vague malgré son utilité évidente. Car quels peuvent être les critères servant à établir qu'une traduction est ou non révisable et jusqu'à quel degré peut-on tolérer les fautes contenues dans un texte? Ces deux questions méritent réflexion.

\subsection{Mossop}

Mossop, quant à lui, propose une série de paramètres beaucoup plus complexes et détaillés. Ceux-ci, au nombre de 12, sont regroupés en quatre domaines: transfert, contenu, langue et présentation. Chaque paramètre se divise, en outre, en plusieurs sous-paramètres:

\begin{tabular}{|l|l|l|l|}
\hline Group 1 & Group 2 & Group 3 & Group 4 \\
\hline Transfer & Content & Language & Presentation \\
\hline Accuracy & Logic & Smoothness & Layout \\
Completeness & Facts & Tailoring & Typography \\
& & Sub-language & Organization \\
& & Idiom & \\
& & Mechanics & \\
\hline
\end{tabular}

Mossop donne l'impression de vouloir mettre l'accent sur l'aspect matériel des textes (présentation, typographie...), ce qui semble refléter un changement de nature pour le travail des réviseurs. Ceux-ci, ne devant plus se contenter de rendre la traduction fidèle au texte original, sont désormais des producteurs de textes à part entière et le sens des responsabilités leur incombe de vérifier si le texte d'arrivée est présentable et autonome.

Mais les 12 paramètres et 32 sous-paramètres pris en compte semblent excessivement nombreux et difficilement applicables. Certains peuvent aussi être assimilés aux critères de l'editing et sujets à récupération quant à l'évaluation de la qualité de textes qui ne sont pas des traductions (voir groupes 3 et 4 ).

\subsection{Synthèse}

Darbelnet a finalement joué un rôle pionnier en proposant pour la première fois des paramètres pour la révision. Certains ont trop été centrés sur les textes spécifiques littéraires ou folkloriques - si bien qu'il n'a abordé le travail du traducteur que sur le plan des mots et des expressions. De plus, certains critères se sont recoupés, rendant 
leur application difficile. Les paramètres d'Horguelin et Brunette, issus en partie de ceux de Darbelnet, sont originaux au sens où lisibilité et rentabilité sont, pour la première fois, pris en compte. Mais aucun moyen concret n'a été proposé pour évaluer la rentabilité dont il est question. Les 12 paramètres de Mossop, classés en quatre groupes, semblent davantage mettre l'accent sur la présentabilité du texte traduit, ce qui nous fait penser que certains de ces paramètres sont plus facilement utilisables pour le travail de l'editing.

Et puisque maints éléments se recoupent et que certains paramètres sont complémentaires, qu'il nous soit permis de proposer les paramètres suivants:

1. Transfert: le message du texte original est-il bien transmis?

2. Norme linguistique: l'usage de la langue d'arrivée est-il respecté?

3. Lisibilité: le texte est-il cohérent, logique et lisible?

4. Adaptation fonctionnelle: le texte prend-t-il en compte la finalité et le destinataire?

\section{Conclusion}

Dans notre étude, nous avons examiné comment le concept de révision a pris forme au cours des dernières décennies et avons constaté que, malgré de nombreuses tentatives définitionnelles, le terme révision reste pluriel et non consensuel. Nous avons donc fait le choix de le définir comme une opération émanant d'une personne connaissant la langue de départ et s'efforçant d'adapter un texte traduit aux besoins du destinataire.

Nous avons ensuite orienté notre réflexion sur les paramètres ciblant l'acte de réviser et susceptibles de garantir un caractère objectif dans le jugement du réviseur. Après avoir étudié les paramètres proposés par Darbelnet, Horguelin et Brunette, puis Mossop, et mis l'accent sur leurs limites, nous avons proposé les nôtres, au nombre de quatre: le transfert de sens, la norme linguistique, la lisibilité et l'adaptation fonctionnelle.

La pluralité des approches relatives à la définition et aux paramètres de la révision reflètent de toute évidence deux choses: d'une part, la complexité du travail du réviseur et, d'autre part, l'insuffisance de recherches cohérentes sur le sujet. La définition et les paramètres que nous proposons ici ont pour but d'élargir le champ de nos recherches aux domaines concernant leur application pédagogique possible, autre sujet d'étude qui pourrait faire l'objet d'une recherche ultérieure.

\section{NOTES}

1. 1) Reading over the entire document. 2) Obtaining background information. 3) Comparing existing translations of the text. 4) Making a first draft of sufficiently comprehensive units. 5) Revising the first draft after a short lapse of time. 6) Reading aloud for style and rhythm. 7) Studying the reactions of receptors by the reading of the text by another person. 8) Submitting a translation to the scrutiny of other competent translators. 9) Revising the text for publication.

2. 1) Dividing the work among members of the Editorial committee. 2) Translating of assigned portions by members of the Editorial Committee. 3) Submitting the work tother members of the Editorial Committee. 4) Studying of these suggestions by the translator. 5) Submitting the resultant draft to the Review Committee. 6) Studying all changes and suggestions made by the Review Committee 7) Preparing a revised draft by the Editorial Secretary. 8) Submitting a revised draft to members of the Consultative Committee. 9) Studying all suggestions made by the Consultative committee. 10) Preparing a final draft. 11) Publishing a final draft. 11) Publishing tentative editions of limited portions. 12) Studying public reaction to limited portions. 13) Polishing of the final draft. 14) Publishing of the complete translation. 
15) Incorporating postpublication corrections into subsequent printings. 16) Postpublication revision of the text.

3. Le comité éditorial propose le premier jet de la traduction, le comité review, composé d'experts, donne son opinion et le comité consultatif approuve ou rejette les versions proposées (Nida 1964: 247).

4. [...] revision implies performing remedial surgery on the submitted text, upgrading the terminology used, clarifying obscurities, reinforcing the impact, honing the emotive appeal to suit the target reader etc. Also included will be consistency of terminology, spelling, grammar, and ensuring that the text is couched in the appropriate language register. [...] (p. 66).

5. D'ailleurs, Didaoui (1998) a considéré le checking et l'editing comme faisant partie des sept types de révision.

\section{RÉFÉRENCES}

Brunette, L. et P. Horguelin (1998): Pratique de la révision, Québec, Linguatec.

Darbelnet, J. (1977): «Niveaux de traduction», Babel 23-1, p. 6-17.

Didaoui, M. (1998): Proceeding of 7th Seminar on Translation Theory and Applications, United Nations Office at Vienna, Vienna International Center.

Graham, J. D. (1989): “Checking, Revision and Editing”, In C. Picken, (Ed.), The Translator's Handbook.

Holmes, J.S. (1972): “The Name and Nature of Translation Studies", In L. Venuti (Ed.), Translation Studies Reader, London, New York, Routledge.

Munday, J. (2001): Introducing Translation Studies: Theories and applications, London and New York, Routledge.

NidA, E. A. (1964): Towards a Science of Translating: With Special Reference to Principles and Procedures Involved in Bible Translating, Leiden, E.J. Brill.

Toury, G. (1995): Descriptive Translation Studies and Beyond, Amsterdam and Philadelphia, John Benjamins. 